International Journal of Difference Equations (IJDE).

ISSN 0973-6069, Volume 15, Number 2, (2020) pp. 215-230

(C) Research India Publications

https://dx.doi.org/10.37622/IJDE/15.2.2020.215-230

\title{
Analytical Solution of Magnetohydrodynamics(MHD) Squeezing Fluid Flow in Porous Medium
}

\author{
Thanon Korkiatsakul $^{1}$, Khomsan Neamprem ${ }^{* 2}$ and Sanoe Koonprasert ${ }^{* 3}$ \\ ${ }^{1}$ Department of Mathematics, Faculty of Science and Technology, \\ Suratthani Rajabhat University, Suratthani 84100, Thailand \\ ${ }^{2}$ Department of Mathematics, Faculty of Applied Science, \\ King Mongkut's University of Technology North Bangkok, \\ Bangkok 10800, Thailand \\ ${ }^{3}$ Department of Mathematics, Faculty of Applied Science, \\ King Mongkut's University of Technology North Bangkok, \\ Bangkok 10800, Thailand
}

\begin{abstract}
The aim of the present work is to compute the magnetohydrodynamic (MHD) squeezing flow through porous medium using Chebyshev wavelet method. Other analytical techniques are compared with the present work. It is shown that results of good agreement can be obtained by choosing a suitable value of convergence control parameter $h$ in the valid region $R_{h}$. The influence of different parameters on the flow is argued theoretically as well as graphically.
\end{abstract}

Keywords: magnetohydrodynamic (MHD), squeezing flow, porous medium, Chebyshev wavelet method.

${ }^{*}$ Corresponding 


\section{INTRODUCTION}

The squeezing of an incompressible viscous fluid between two parallel plates is a fundamental type of flow that is frequently observed in many hydrodynamical tools and machines. Compression and injection molding, polymer processing, and modeling of lubrication systems are some practical examples of squeezing flows where their usage is found. The modeling and analysis of squeezing flow have been started in the nineteenth century and continues to receive significant attention due to its vast application areas in biophysical and physical science [1,6,11,16].

Magnetohydrodynamics (MHD) also magneto fluid dynamics or hydromagnetic is the study of the magnetic properties of electrically conducting fluids, examples of such magneto fluids include plasmas, liquid metals, salt water, and electrolytes. The fundamental concept behind MHD is that magnetic fields can induce currents in a moving conductive fluid, which in turn polarizes the fluid and reciprocally changes the magnetic field itself. The set of equations that describe MHD are a combination of the Navier-Stokes equations of fluid dynamics and Maxwell's equations of electromagnetism. These differential equations must be solved simultaneously, either analytically or numerically $[7,9,10,17]$.

The simplest form of MHD, assumes that the fluid has so little resistivity that it can be treated as a perfect conductor. To explain in ideal MHD a small rope-like volume of fluid surrounding a field line will continue to lie along a magnetic field line, even as it is twisted and distorted by fluid flows in the system. The connection between magnetic field lines and fluid in ideal MHD fixes the topology of the magnetic field in the fluid, for example, if a set of magnetic field lines are tied into a knot, then they will remain so as long as the fluid/plasma has negligible resistivity. This difficulty in reconnecting magnetic field lines makes it possible to store energy by moving the fluid or the source of the magnetic field. The energy can then become available if the conditions for ideal MHD break down, allowing magnetic reconnection that releases the stored energy from the magnetic field $[10,12]$.

The study would be useful in optimizing energy usage and other benefits. A literature search reveals that the problem has remained a hot topic among the researchers who have studied the flow and heat transfer through a porous medium in both open and closed regions. Moreover, both conventional fluids and nanofluids have been utilized during such diverse studies. The present study will stick to the cavity flow with conventional fluid. The results were validated with the reported experimental results. In another study, the flow through the wavy vertical-cavity filled with the porous medium was considered. The solution was obtained numerically using the Finite Element Method. Sheremet et al. incorporated nanofluid in Buongiorno's model to study the three dimensional natural convection heat transfer in a cubical cavity filled with a porous medium $[2,3,15]$. 
In Chebyshev wavelet method, there are several advantages of using Chebyshev wavelets approximations, unlike most analytical methods, it is now conventional that they are characterized by the use of exponential errors. Any numerical methods do not perform well near singularities, whereas approximations through wavelets effectively handle singularities in the problem. Due to their fast convergence, Chebyshev wavelets method does not undergo from the instability problems related to other numerical methods. In 2013, Y. Chen et al. proposed the Chebyshev wavelet method by solving fractional integral and differential equations of Bratu-type [5]. In 2015, A.K. Gupta and S.S. Ray studied the solution of fractional fifth-order Sawada-Kotera equation using second kind Chebyshev wavelet method [8]. In 2019, T. Korkiatsakul et al. proposed the Chebyshev wavelet method by solving the generalized time fractional KolmogorovPetrovsky-Piskunov equation (FKPP) with variety of initial-boundary conditions [13].

This work is an effort to investigate MHD squeezing flow of Newtonian fluid between two parallel plates passing through porous medium. The governing partial differential equations are in equivalent nonlinear ordinary differential equation and then solved using the mentioned Chebyshev wavelet scheme. Velocity profile of fluid is argued by varying various parameters involved.

\section{MATHEMATICAL MODEL}

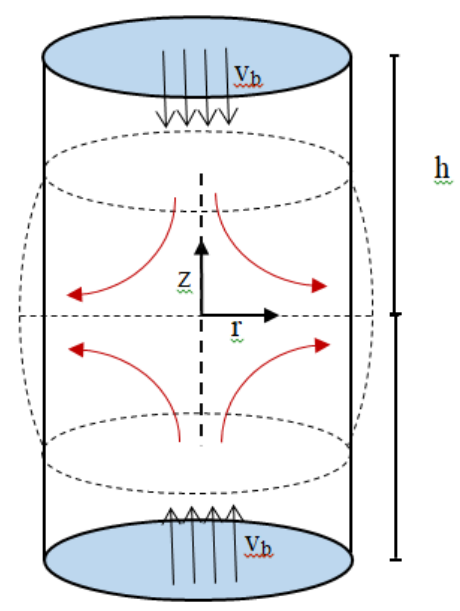

Figure 2.1: A steady squeezing axisymmetric fluid flow between two parallel plates.

We consider the presence of a magnetic field, a squeezing flow of an incompressible Newtonian fluid with constant density $\rho$, and viscosity $\mu$, squeezed between two large planar parallel plates separated by a small distance $2 h$ approaching each other with a low constant velocity $\mathbf{v}$ in Fig.2.1. Assume that the flow is quasisteady, and the Navier- 
Stokes equations governing such flow when inertial terms are retained as

$$
\begin{aligned}
\nabla \cdot \mathbf{v} & =0, \\
\rho\left[\frac{\partial \mathbf{v}}{\partial t}+(\nabla \cdot \mathbf{v}) \mathbf{v}\right] & =\nabla \cdot \mathbf{T}+(\mathbf{v} \times \mathbf{B}) \times \mathbf{B},
\end{aligned}
$$

where $\nabla$ denotes the material time derivative, $\mathbf{T}$ is the Cauchy stress tensor given by $\mathbf{T}=-p I+\mu A$ with $A=\nabla \mathbf{v}+(\mathbf{v})^{t}$. B is the total magnetic field given by $\mathbf{B}=\mathbf{B}_{0}+b$. $\mathbf{B}_{0}$ and $b$ represent the imposed and induced magnetic fields, respectively. The modified Ohm's law and Maxwell's equations, in the absence of displacement currents, are

$$
\begin{array}{cr}
\mathrm{J}=\sigma[E+\mathbf{v} \times \mathbf{B}], & \nabla \cdot \mathbf{B}=0, \\
\nabla \times \mathbf{B}=\mu_{m} \mathrm{~J}, & \operatorname{curl} E=\frac{\partial \mathbf{B}}{\partial t} .
\end{array}
$$

Here $\mathrm{J}$ is the electric current density, $\sigma$ represents the electrical conductivity, $E$ the electric field, and $\mu_{m}$ the magnetic permeability. If $\rho, \mu_{m}$, and $\sigma$ are constant, $b$ is negligible as compared to $\mathbf{B}_{0}, \mathbf{B}$ is perpendicular to $\mathbf{v}$ so that the Reynold number is small with no electric field in the fluid flow region and then the magnetohydrodynamic force involved can be written as

$$
\mathrm{J} \times \mathbf{B}=-\sigma \mathbf{B}_{0}^{2} \mathbf{v}
$$

The plates are nonconducting and the magnetic field is applied along the $z$-axis. The gap distance $2 h$ between the plates changes slowly with time $t$ for small values of the velocity $\mathbf{v}$ so that it can be taken as constant. An axisymmetric flow in cylindrical coordinates $(r, \theta, z)$ with $z$-axis perpendicular to plates and $z= \pm h$ at the plates. For axial symmetry, $\mathbf{v}$ is represented by $\mathbf{v}=\left(v_{r}, 0, v_{z}\right)$. If $v_{r}^{2}+v_{z}^{2}=u$, then from (2.1), we have

$r$ component:

$$
\frac{\partial}{\partial r}(\rho u+p)-\rho \Omega v_{z}=-\left(\mu \frac{\partial \Omega}{\partial z}+A v_{r}\right)
$$

$\theta$ component:

$$
\frac{1}{r} \frac{\partial p}{\partial \theta}=0 \Longrightarrow \frac{\partial p}{\partial \theta}=0 \Longrightarrow p=f(r, z)
$$

$z$ component:

$$
\frac{\partial}{\partial z}(\rho u+p)-\rho \Omega v_{r}=\frac{\mu}{r} \frac{\partial}{\partial r}(r \Omega)-\frac{\mu}{\kappa} v_{z}
$$

where

$$
A=\frac{\mu}{\kappa}+\sigma B_{0}^{2}, \quad \Omega(r, z)=\frac{\partial v_{z}}{\partial r}-\frac{\partial v_{r}}{\partial z} .
$$

Introducing stream function $\phi(r, z)$ as defined by 


$$
v_{r}(r, z)=\frac{1}{r} \frac{\partial \phi}{\partial z}, \quad v_{z}(r, z)=-\frac{1}{r} \frac{\partial \phi}{\partial r} .
$$

They satisfy the continuity equation $\nabla \cdot \mathbf{v}=0$. With the help of (2.6) and the generalized pressure $\hat{p}=\rho u+p$, from (2.2) and (2.4), we have:

$$
\begin{aligned}
\rho\left[\frac{\partial \phi}{\partial r}\right. & \left.\frac{\partial}{\partial z}\left(\frac{\eta^{2} \phi}{r^{2}}\right)-\frac{\partial \phi}{\partial z} \frac{\partial}{\partial r}\left(\frac{\eta^{2} \phi}{r^{2}}\right)\right] \\
= & -\frac{\mu}{r}\left[\frac{\partial^{4}}{\partial r^{4}}-\frac{2}{r} \frac{\partial^{3}}{\partial r^{3}}+\frac{1}{r^{2}} \frac{\partial^{2}}{\partial r^{2}}-\frac{3}{r^{3}} \frac{\partial}{\partial r}+2 \frac{\partial^{4}}{\partial r^{2} \partial z^{2}}\right. \\
& \left.-\frac{2}{r} \frac{\partial^{3}}{\partial r \partial z^{3}} \frac{\partial^{4}}{\partial z^{4}}\right] \phi+\frac{\mu}{\kappa r} \eta^{2} \phi+\frac{\sigma B_{0}^{2}}{r} \frac{\partial^{2} \phi}{\partial z^{2}} \\
= & -\frac{\mu}{r} \eta^{4} \phi+\frac{\mu}{\kappa r} \eta^{2} \phi+\frac{\sigma B_{0}^{2}}{r} \frac{\partial^{2} \phi}{\partial z^{2}},
\end{aligned}
$$

where, $\eta^{2}=\frac{\partial^{2}}{\partial r^{2}}-\frac{1}{r} \frac{\partial}{\partial r}+\frac{\partial^{2}}{\partial z^{2}}$. Since the moving plates are separated by distance $2 d$, then

$$
v_{r}(r, d)=0, v_{z}(r, d)=-u, v_{z}(r, 0)=0, \frac{\partial v_{r}(r, 0)}{\partial z}=0 .
$$

Using the transformation $\phi(r, z)=r^{2} f(z)$, (2.7) can reduce into the ordinary differential equation as follows:

$$
\begin{gathered}
2 \rho r f(z) \frac{d^{3}}{d z^{3}} f(z)=-\mu r \frac{d^{4}}{d z^{4}} f(z)+\frac{\mu}{\kappa} r \frac{d^{2}}{d z^{2}} f(z)+\sigma B_{0}^{2} r \frac{d^{2}}{d z^{2}} f(z), \\
\frac{d^{4}}{d z^{4}} f(z)+\frac{2 \rho}{\mu} f(z) \frac{d^{3}}{d z^{3}} f(z)-\frac{1}{\kappa} \frac{d^{2}}{d z^{2}} f(z)-\frac{\sigma B_{0}^{2}}{\mu} \frac{d^{2}}{d z^{2}} f(z)=0 .
\end{gathered}
$$

The boundary conditions in (2.8) are transformed by

$$
\frac{d^{2}}{d z^{2}} f(0)=0, f(0)=0 \frac{d}{d z} f(d)=0, f(d)=-\frac{u}{2} .
$$

Applying nondimensional parameters as $\frac{2}{u} f^{*}=f, d z^{*}=d z, \frac{u}{\mu} R_{m p}=\rho d, m_{h}=$ $\frac{\sigma d B_{0}^{2}}{\mu}$, and $m_{p}=\frac{h}{\kappa}$ (omitting $*$ sign), (2.9) and (2.10) become

$$
\frac{d^{4}}{d z^{4}} f(z)+R_{m p} f(z) \frac{d^{3}}{d z^{3}} f(z)-m_{p} \frac{d^{2}}{d z^{2}} f(z)-m_{h} \frac{d^{2}}{d z^{2}} f(z)=0,
$$

with the boundary conditions:

$$
\frac{d^{2}}{d z^{2}} f(0)=0, f(0)=0, \frac{d}{d z} f(1)=0, f(1)=1,
$$

where $R_{m p}$ is Reynold and $m_{h}, m_{p}$ are Hartmann numbers. 


\section{PRELIMINARIES}

In this section, we introduce some necessary definitions of variety Chebyshev wavelets.

\subsection{Chebyshev wavelets function}

Wavelets have been very successful in many sciences and constitute a family of functions constructed from dilation and translation of a single function called the mother wavelet $\psi(z)$. When the dilation parameter $a$ and the translation parameter $b$ vary continuously, we have the following family of continuous wavelets

$$
\psi_{a, b}(z)=|a|^{\frac{1}{2}} \psi\left(\frac{z-b}{a}\right), \quad a, b \in \mathbb{R}, a \neq 0 .
$$

Chebyshev wavelets $\psi_{n, m}(z)=\psi(k, 2 n-1, m, z)$ have three arguments; $k \in N, n=$ $1,2, \ldots, 2^{k-1}$, and $m$ is the degree of the Chebyshev polynomial of the first kind where $z \in[0,1)$. They are defined on the interval $[0,1)$ as $[4,14]$

$$
\psi_{n, m}(z)= \begin{cases}\alpha_{m} 2^{k-1 / 2} T_{m}\left(2^{k} z-2 n+1\right), & \\ & \frac{n-1}{2^{k-1}} \leq t<\frac{n}{2^{k-1}} \\ 0, & \text { Otherwise, }\end{cases}
$$

where

$$
\alpha_{m}= \begin{cases}\sqrt{2}, & m=0, \\ 2, & m=1,2, \ldots\end{cases}
$$

and $m=0,1,2, \ldots, M-1, n=1,2, \ldots, 2^{k-1}$. Here, $T_{m}(z)$ are the set of well-known Chebyshev polynomials of order $m$, which are orthogonal with respect to the weight function $\omega(z)=\frac{1}{\sqrt{1-z^{2}}}$ and they satisfy the following recursive formula:

$$
\begin{aligned}
T_{0}(z) & =1, \quad T_{1}(z)=z, \\
T_{m+1}(z) & =2 z T_{m}(z)-T_{m-1}(z), \quad m=1,2, \ldots
\end{aligned}
$$

We should note that the set of Chebyshev wavelets is orthogonal with respect to the weight function $\omega_{n}(z)=\omega\left(2^{k} z-2 n+1\right)$. The derivative of Chebyshev polynomials is a linear combination of lower-order Chebyshev polynomials, in fact,

$$
T_{m}^{\prime}(z)= \begin{cases}2 m \sum_{k=1}^{m-1} T_{m}(z), & m \text { even } \\ 2 m \sum_{k=1}^{m-1} T_{m}(z)+m T_{0}(z), & m \text { odd } .\end{cases}
$$




\subsection{Chebyshev wavelet operational matrix}

A function $f(z)$ defined over interval $[0,1)$ can expand with Chebyshev wavelets as

$$
f(z)=\sum_{n=1}^{\infty} \sum_{m=0}^{\infty} c_{n, m} \psi_{n, m}(z),
$$

If the infinite series in (3.3) is truncated, then it can be written as

$$
f(z) \simeq \sum_{n=1}^{2^{k-1}} \sum_{m=0}^{M-1} c_{n, m} \psi_{n, m}(z)=C \Psi(z)
$$

where $C$ and $\Psi(z)$ are $2^{k-1} M$ column vectors that given by

$$
\begin{aligned}
C= & {\left[c_{10}, c_{11}, \ldots, c_{1(M-1)}, c_{20}, c_{21}, \ldots, c_{2(M-1)}, \ldots,\right.} \\
& \left.c_{2^{k-1} 0}, c_{2^{k-1}}, \ldots, c_{2^{k-1}(M-1)}\right] \\
\Psi(z)= & {\left[\psi_{10}, \psi_{11}, \ldots, \psi_{1(M-1)}, \psi_{20}, \psi_{21}, \ldots, \psi_{2(M-1)},\right.} \\
& \left.\ldots, \psi_{2^{k-1} 0}, \psi_{2^{k-1} 1}, \ldots, \psi_{2^{k-1}(M-1)}\right]^{T}
\end{aligned}
$$

\subsection{Chebyshev wavelets operational matrix of derivative}

In this section, we first derive the operational matrix $D$ of derivative. In the interval $\left[\frac{n-1}{2^{k-1}}, \frac{n}{2^{k-1}}\right)$,

$$
\psi_{n, m}(z)=\frac{\alpha_{m} 2^{\frac{k-1}{2}}}{\sqrt{\pi}} T_{m}\left(2^{k} z-2 n+1\right) .
$$

Applying (3.6) the derivative of $\psi_{n, m}(z)$ is

$$
\psi_{n, m}^{\prime}(z)=\left\{\begin{array}{c}
\frac{\alpha_{m} 2^{(k-1) / 2}}{\sqrt{\pi}} \cdot 2^{k} \cdot 2 m \sum_{k=1}^{m-1} T_{k}\left(2^{k} z-2 n+1\right), \\
\frac{\alpha_{m} 2^{(k-1) / 2}}{\sqrt{\pi}} \cdot 2^{k} \cdot\left[2 m \sum_{k=1}^{m-1} T_{k}\left(2^{k} z-2 n+1\right)\right. \\
\left.+m T_{0}\left(2^{k} z-2 n+1\right)\right], \quad m \text { odd } .
\end{array}\right.
$$

The function $\psi_{n, i}(z)$ is zero outside the interval $\left[\frac{i-1}{2^{k-1}}, \frac{i}{2^{k-1}}\right)$ so

$$
\psi_{n, i}^{\prime}(z)=F \psi_{n, i}(z), \quad i=1,2, \ldots, 2^{k-1},
$$


where

$$
F=2^{k} \cdot\left[\begin{array}{ccccc}
0 & 0 & \cdots & 0 & 0 \\
\sqrt{2} & 0 & \cdots & 0 & 0 \\
0 & 4 & \cdots & 0 & 0 \\
3 \sqrt{2} & 0 & \cdots & 0 & 0 \\
0 & 8 & \cdots & 0 & 0 \\
5 \sqrt{2} & 0 & \cdots & 0 & 0 \\
\vdots & \vdots & \ddots & \vdots & 0 \\
(M-1) \sqrt{2} & 0 & \cdots & 2(M-1) & 0
\end{array}\right]_{M \times M}
$$

for even $M$,

$$
F=2^{k} \cdot\left[\begin{array}{cccccc}
0 & 0 & 0 & \cdots & 0 & 0 \\
\sqrt{2} & 0 & 0 & \cdots & 0 & 0 \\
0 & 4 & 0 & \cdots & 0 & 0 \\
3 \sqrt{2} & 0 & 6 & \cdots & 0 & 0 \\
0 & 8 & 0 & \cdots & 0 & 0 \\
5 \sqrt{2} & 0 & 10 & \cdots & 0 & 0 \\
\vdots & \vdots & \vdots & \ddots & \vdots & 0 \\
0 & 0 & 2(M-1) & \cdots & 2(M-1) & 0
\end{array}\right]_{M \times M}
$$

for odd $M$.

In fact we have shown that

$$
\frac{d \Psi(z)}{d z}=D \Psi(z)
$$

where

$$
D=\left[\begin{array}{cccc}
F & 0 & \ldots & 0 \\
0 & F & \ldots & 0 \\
\vdots & \vdots & \ddots & \vdots \\
0 & 0 & \ldots & F
\end{array}\right]
$$

From (3.9), it can be generalized for any $n \in \mathbb{N}$ as

$$
\frac{d^{n} \Psi(z)}{d z^{n}}=D^{n} \Psi(z), \quad n=1,2,3, \ldots
$$

\section{CHEBYSHEV SOLUTION FOR MHD SQUEEZING FLOW IN POROUS MEDIUM}

In this section, the nondimensional of Magnetohydrodynamic Squeezing Flow in Porous Medium (2.11) and boundary conditions (2.12)

$$
\frac{d^{4}}{d z^{4}} f(z)+R_{m p} f(z) \frac{d^{3}}{d z^{3}} f(z)-m_{p} \frac{d^{2}}{d z^{2}} f(z)-m_{h} \frac{d^{2}}{d z^{2}} f(z)=0,
$$


and the boundary conditions:

$$
\frac{d^{2}}{d z^{2}} f(0)=0, f(0)=0, \frac{d}{d z} f(1)=0, f(1)=1 .
$$

We employ the solution of the model by the Chebyshev wavelets, we first approximate $f(z)$ as

$$
f(z)=C \Psi(z)
$$

Applying (3.11) we can get

$$
\frac{d^{2}}{d z^{2}} f(z)=C D^{2} \Psi(z), \frac{d^{3}}{d z^{3}} f(z)=C D^{3} \Psi(z) \text { and } \frac{d^{4}}{d z^{4}} f(z)=C D^{4} \Psi(z) .
$$

From (4.1), we have

$$
C D^{4} \Psi(z)+R_{m p}(C \Psi(z))\left(C D^{3} \Psi(z)\right)-m_{p}\left(C D^{2} \Psi(z)\right)+m_{h}\left(C D^{2} \Psi(z)\right)=0 .
$$

Suitable collocation points are

$$
z_{i}=\frac{2 i-1}{2^{k} M}, \quad i=1,2, \ldots, 2^{k-1} M .
$$

We now collocate (4.2) at $2^{k-1} M$ points at $z_{i}$ as the system

$$
C D^{4} \Psi\left(z_{i}\right)+R_{m p}\left(C \Psi\left(z_{i}\right)\right)\left(C D^{3} \Psi\left(z_{i}\right)\right)-m_{p}\left(C D^{2} \Psi\left(z_{i}\right)\right)+m_{h}\left(C D^{2} \Psi\left(z_{i}\right)\right)=0,
$$

with the boundary conditions

$$
C \Psi(0)=0, C \Psi(1)=1, C D^{2} \Psi(0)=0, C D \Psi(1)=0 .
$$

Equations (4.3) and (4.4) generate $2^{k-1} M$ set of nonlinear equations. The vector $C$ is obtained by solving the nonlinear system using the Gauss-Newton method.

\section{ERROR ANALYSIS}

In this section we present the error analysis.

Theorem 5.1. A function $f(z) \in L_{\omega}^{2}([0,1])$, with bounded second derivative, say $\left|\frac{d^{4}}{d z^{4}} f(z)\right| \leq N, N$ is integer can be expanded as an infinite sum of Chebyshev wavelets, and the series converges uniformly to $f(z)$, that is,

$$
f(z)=\sum_{n=1}^{\infty} \sum_{m=0}^{\infty} c_{n m} \psi_{n m} .
$$


Since the truncated Chebyshev wavelets series, $\widehat{f}(z)=C \Psi(z)$ is an approximate solution of Magnetohydrodynamic Squeezing Flow in Porous Medium, so one has an error function $E(z)$ for $f(z)$ as follows:

$$
E(z)=|f(z)-\widehat{f}(z)|
$$

The error bound of the approximate solution by using Chebyshev wavelets series is given by the following theorem.

Theorem 5.2. Suppose that $f(z) \in C^{m}[0,1]$ and $\widehat{f}(z)=C \Psi(z)$ is the approximate Chebyshev wavelet solution using the Chebyshev wavelets method. Then the error bound would be obtained as follows:

$$
E(z) \leq \frac{2}{m ! 4^{m} 2^{m(k-1)}} \max _{z \in[0,1]}\left|f^{m}(z)\right|
$$

Proof. The interval $[0,1]$ is divided into $2^{k-1}$ subintervals $I_{n}=\left[\frac{n-1}{2^{k-1}}, \frac{n}{2^{k-1}}\right]$, $n=1,2, \ldots, 2^{k-1}$. The interpolating polynomial, $P_{m}(z)$, of degree $m$ which agrees with $\widehat{f}(z)=C \Psi(z)$ at the Chebyshev nodes on $I_{n}$ with the error bound for interpolating

$$
\left|f(z)-P_{m}(z)\right| \leq \frac{2}{m ! 4^{m} 2^{m(k-1)}} \max _{z \in[0,1]}\left|f^{m}(z)\right| .
$$

By the norm of the inner product space, we have

$$
\begin{aligned}
\|E(z)\|^{2} & =\|f(z)-\widehat{f}(z)\| \\
& =\int_{0}^{1}(f(z)-\widehat{f}(z))^{2} d z \\
& \leq \sum_{n=1}^{2^{k-1}} \int_{\frac{n-1}{2^{k-1}}}^{\frac{n}{2^{k-1}}}(f(z)-\widehat{f}(z))^{2} d z \\
& \leq \sum_{n=1}^{2^{k-1}} \int_{\frac{n-1}{2^{k-1}}}^{\frac{n}{2^{k-1}}}\left|f(z)-P_{m}(z)\right|^{2} d z, \\
& \leq \sum_{n=1}^{2^{k-1}} \int_{\frac{n-1}{2^{k-1}}}^{\frac{n}{2^{k-1}}}\left(\frac{2}{m ! 4^{m} 2^{m(k-1)}} \max _{z \in[0,1]}\left|f^{m}(z)\right|\right)^{2} d z \\
& =\left(\frac{2}{m ! 4^{m} 2^{m(k-1)}} \max _{z \in[0,1]}\left|f^{m}(z)\right|\right)^{2} \sum_{n=1}^{2^{k-1}} \int_{\frac{n-1}{2^{k-1}}}^{\frac{n}{2^{k-1}}} d z \\
& =\left(\frac{2}{m ! 4^{m} 2^{m(k-1)}} \max _{z \in[0,1]}\left|f^{m}(z)\right|\right)^{2}
\end{aligned}
$$




\section{NUMERICAL RESULTS}

In this section, we implement the Chebyshev wavelets for the magnetohydrodynamic (MHD) squeezing flow through porous medium.

Example Consider a special case of Magnetohydrodynamic Squeezing Flow in Porous Medium when the Reynold number is zero $\left(R_{m p}=0\right)$ :

$$
\frac{d^{4}}{d z^{4}} f(z)-m_{p} \frac{d^{2}}{d z^{2}} f(z)-m_{h} \frac{d^{2}}{d z^{2}} f(z)=0,
$$

and the boundary conditions:

$$
\frac{d^{2}}{d z^{2}} f(0)=0, f(0)=0, \frac{d}{d z} f(1)=0, f(1)=1 .
$$

The exact solution is given by

$$
\begin{aligned}
f(z)= & \frac{\sqrt{M} z}{\sqrt{M} e^{2 \sqrt{M}}+\sqrt{M}-e^{2 \sqrt{M}}+1}+\frac{\sqrt{M} z e^{2 \sqrt{M}}}{\sqrt{M} e^{2 \sqrt{M}}+\sqrt{M}-e^{2 \sqrt{M}}+1} \\
& -\frac{e^{\sqrt{M} z+\sqrt{M}}-e^{\sqrt{M}}}{\sqrt{M} e^{2 \sqrt{M}}+\sqrt{M}-e^{2 \sqrt{M}}+1}
\end{aligned}
$$

where $M=m_{h}+m_{p}$.

The Chebyshev wavelet solution where $k=1, M=4$ is given by

$$
\begin{aligned}
f(z)= & 0.7441552691 \frac{\sqrt{2}}{\sqrt{\pi}}+0.9139215170 \frac{2 z-1}{\sqrt{\pi}}-0.1661675485 \frac{8 z^{2}-8 z+1}{\sqrt{\pi}} \\
& -0.02769459142 \frac{32 z^{3}-48 z^{2}+18 z-1}{\sqrt{\pi}} .
\end{aligned}
$$

For $k=2, M=6$ the Chebyshev wavelet solution is

$$
\begin{aligned}
f(z)= & 0.6226858440 \frac{1}{\sqrt{\pi}}+0.429047997 \frac{\sqrt{2}(4 z-1)}{\sqrt{\pi}} \\
& -0.01354226207 \frac{\sqrt{2}\left(32 z^{2}-16 z+1\right)}{\sqrt{\pi}} \\
& -0.002300643 \frac{\sqrt{2}\left(256 z^{3}-192 z^{2}+36 z-1\right)}{\sqrt{\pi}} \\
& -0.000017556 \frac{\sqrt{2}\left(2048 z^{4}-\ldots-64 z+1\right)}{\sqrt{\pi}} \\
& -0.00000179 \frac{\sqrt{2}\left(16384 z^{5}-\ldots+100 z-1\right)}{\sqrt{\pi}} .
\end{aligned}
$$

Tables 6.1 and 6.2 show the comparison of Chebyshev wavelet solution and the exact solution and the absolute errors. The numerical results obtained from evaluating Chebyshev wavelet method have high accuracy as the following tables with $(k=1, M=4)$ and $(k=2, M=6)$. 
Table 6.1: Exact, Chebyshev Wavelet solution where $k=1, M=4$ and Absolute errors

\begin{tabular}{cccc}
$\mathrm{z}$ & Exact solutions & $\mathrm{CW}$ solutions & Absolute errors \\
\hline 0.0 & 0.0 & $2 \times 10^{-10}$ & $2 \times 10^{-10}$ \\
0.1 & 0.1471713482 & 0.1494999998 & 0.0023286516 \\
0.2 & 0.2916176128 & 0.2959999998 & 0.0043823871 \\
0.3 & 0.4305864345 & 0.4364999998 & 0.0059135653 \\
0.4 & 0.5612706364 & 0.5679999998 & 0.0067293632 \\
0.5 & 0.6807801247 & 0.6874999998 & 0.0067198747 \\
0.6 & 0.7861129669 & 0.7919999998 & 0.0058870328 \\
0.7 & 0.8741253420 & 0.8784999998 & 0.0043746578 \\
0.8 & 0.9415000850 & 0.9439999998 & 0.0024999143 \\
0.9 & 0.9847134817 & 0.9854999998 & 0.0007865178 \\
1.0 & 1.0 & 0.9999999999 & $1 \times 10^{-10}$
\end{tabular}

Table 6.2: Exact, Chebyshev Wavelet solution where $k=2, M=6$ and Absolute errors

\begin{tabular}{cccc}
$\mathrm{z}$ & Exact solutions & CW solutions & Absolute errors \\
\hline 0.0 & 0.0 & 0.0 & 0.0 \\
0.1 & 0.1471713482 & 0.1471713488 & 0.0000000006 \\
0.2 & 0.2916176128 & 0.2916176131 & 0.0000000003 \\
0.3 & 0.4305864345 & 0.4305864350 & 0.0000000005 \\
0.4 & 0.5612706364 & 0.5612706369 & 0.0000000005 \\
0.5 & 0.6807801247 & 0.6807801254 & 0.0000000007 \\
0.6 & 0.7861129669 & 0.7861129666 & 0.0000000003 \\
0.7 & 0.8741253420 & 0.8741253427 & 0.0000000007 \\
0.8 & 0.9415000850 & 0.9415000865 & 0.0000000015 \\
0.9 & 0.9847134817 & 0.9847134824 & 0.0000000007 \\
1.0 & 1.0 & 1.0 & 0.0
\end{tabular}


For figures 6.1 and 6.2 we can show the graph of Chebyshev wavelet solution and the exact solution with $k=1, M=4$ and $k=2, M=6$.

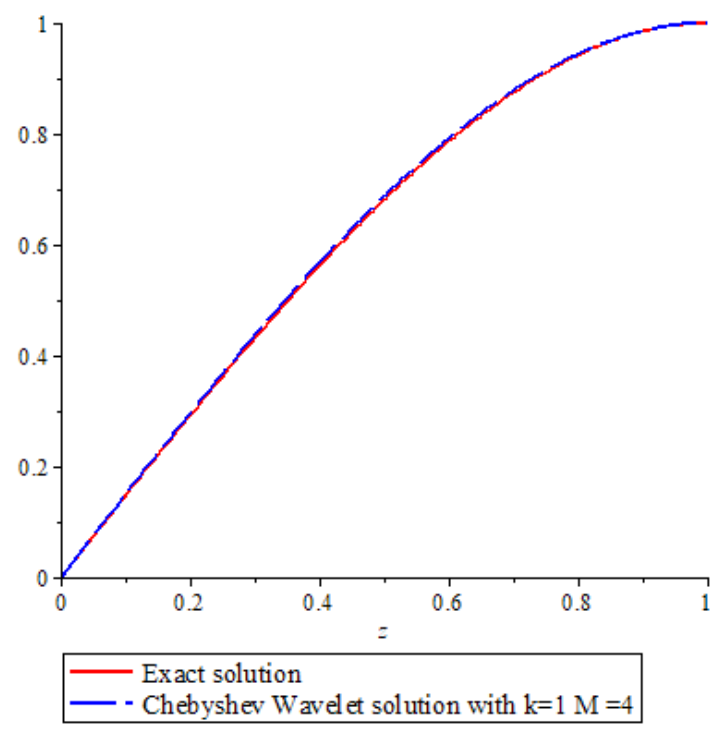

Figure 6.1: Graph of Exact solution and Chebyshev wavelet solutions where $k=1, M=4$.

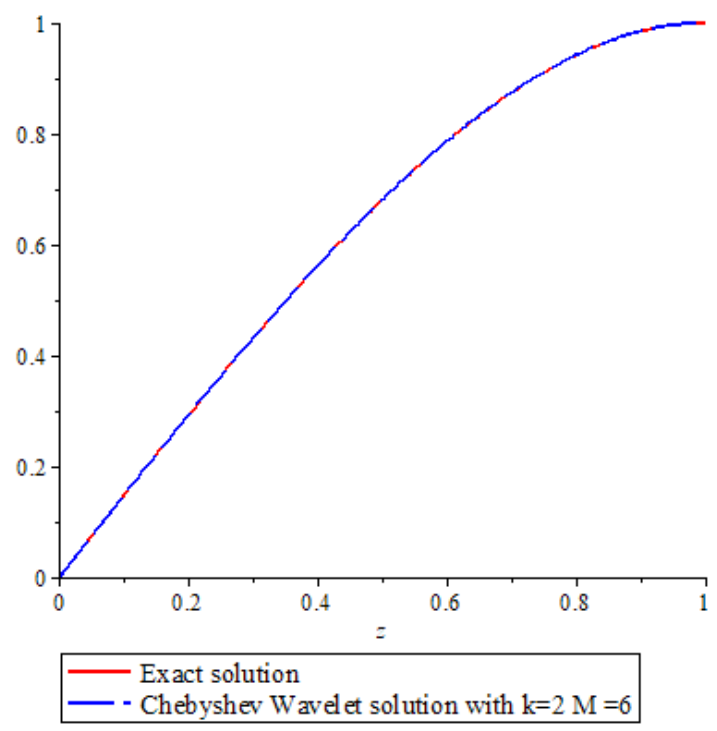

Figure 6.2: Graph of Exact solution and Chebyshev wavelet solutions where $k=2, M=6$.

From $\psi(r, z)=r^{2} f(z)$ we can plot the graph of $\psi(r, z)$ as 

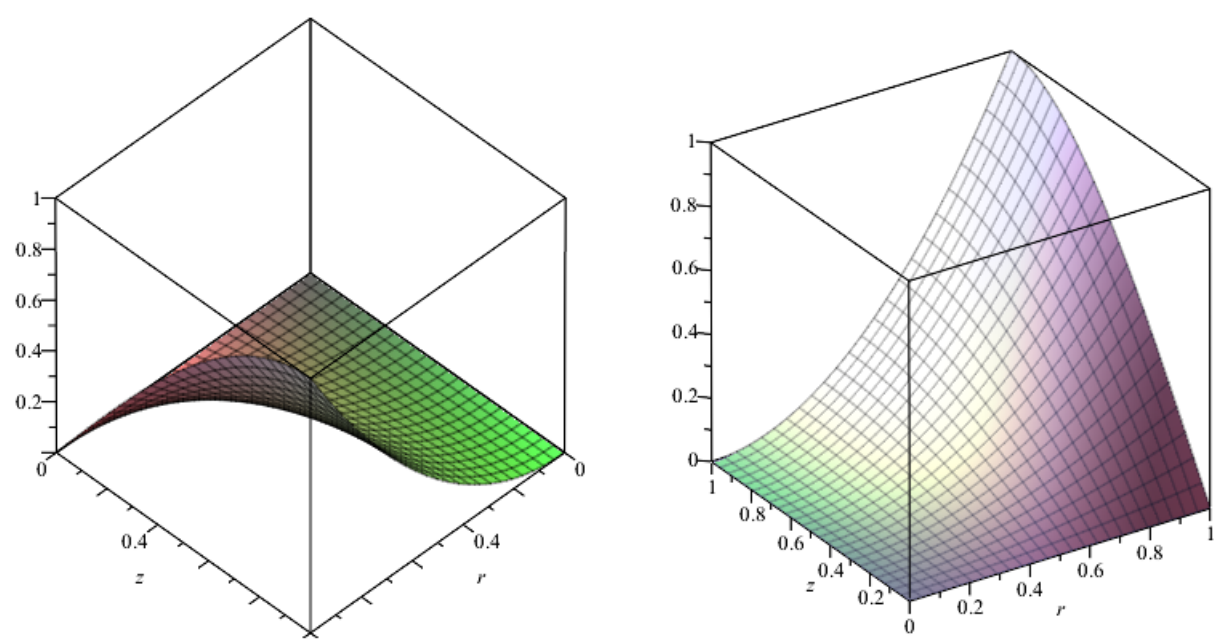

Figure 6.3: Graph of Chebyshev solution of $\psi(r, z)$.

Introducing stream function $\psi(r, z)$ defined as

$$
v_{r}(r, z)=\frac{1}{r} \frac{\partial \psi}{\partial z}, \quad v_{z}(r, z)=-\frac{1}{r} \frac{\partial \psi}{\partial r}
$$

then we can plot the graph of velocity $r$ component and $z$ component as
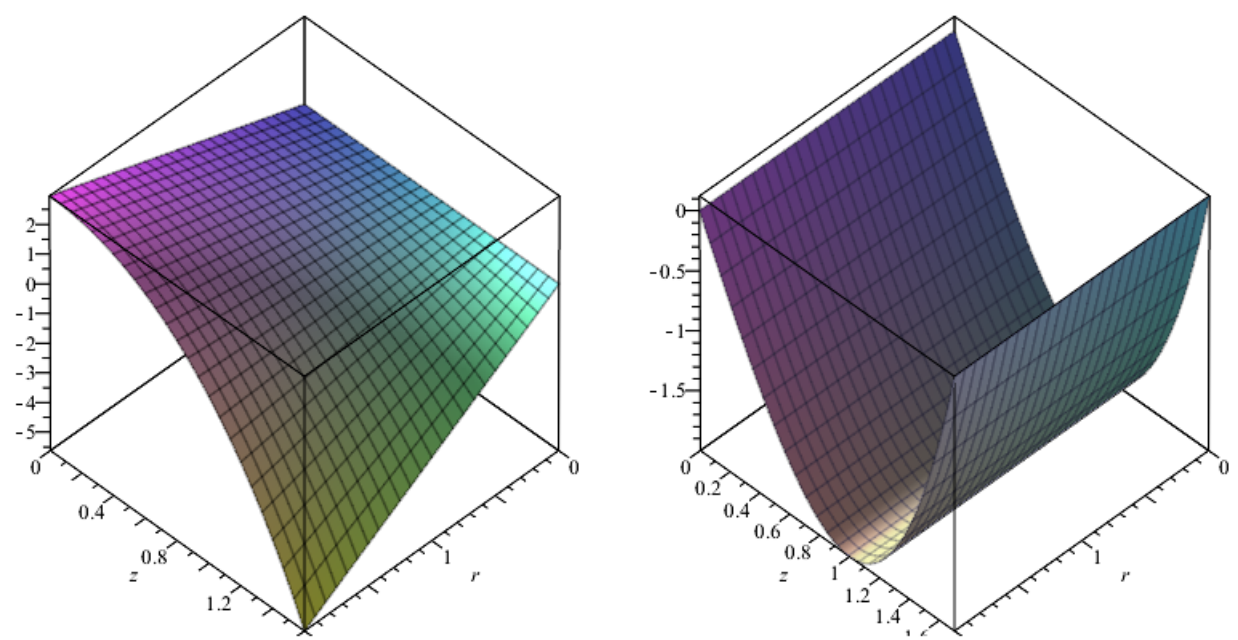

Figure 6.4: Graph of Velocity $r$ components and $z$ components.

\section{CONCLUSIONS}

In this paper, the Chebyshev wavelet method is applied to solve initial-boundary value problems of the differential equation which application for a magnetohydrodynamic (MHD) squeezing flow through porous medium. This method is simple and a good 
mathematical method for finding analytical solutions of differential equation. The validity, accuracy and applicability of our Chebyshev wavelet method have been illustrated through numerical results by showing the absolute errors between an exact solution and Chebyshev wavelet solutions in Table 6.1 and accuracy and efficiency of our method are reported by the absolute errors in Table 6.2. Moreover, Chebyshev wavelet technique is powerful method for solving differential equations.

\section{Acknowledgment}

We would like to thank the Department of Mathematics, Faculty of Applied Science, King Mongkut's University of Technology North Bangkok, Thailand and Department of Mathematics, Faculty of Science and Technology, Suratthani Rajabhat University, Thailand for supporting us in doing this work. The second author was financially supported by the Faculty of Applied Science, King Mongkut's University of Technology North Bangkok under contract no. 6242105.

\section{REFERENCES}

[1] Maryam Aleem, Muhammad Imran Asjad, Aqila Shaheen, and Ilyas Khan. Mhd influence on different water based nanofluids (tio2, al2o3, cuo) in porous medium with chemical reaction and newtonian heating. Chaos, Solitons \& Fractals, 130:109437, 2020.

[2] Fahd Almutairi, SM Khaled, and Abdelhalim Ebaid. Mhd flow of nanofluid with homogeneous-heterogeneous reactions in a porous medium under the influence of second-order velocity slip. Mathematics, 7(3):220, 2019.

[3] Talha Anwar, Ilyas Khan, Poom Kumam, and Wiboonsak Watthayu. Impacts of thermal radiation and heat consumption/generation on unsteady mhd convection flow of an oldroyd-b fluid with ramped velocity and temperature in a generalized darcy medium. Mathematics, 8(1):130, 2020.

[4] Jafar Biazar and H Ebrahimi. Chebyshev wavelets approach for nonlinear systems of volterra integral equations. Computers \& Mathematics with Applications, 63(3):608-616, 2012.

[5] YM Chen, Lu Sun, LL Liu, and JQ Xie. The chebyshev wavelet method for solving fractional integral and differential equations of bratu-type. J. Comput. Inf. Syst, 9(14):5601-5609, 2013.

[6] Christian Geindreau and JL Auriault. Magnetohydrodynamic flows in porous media. Journal of fluid mechanics, 466:343, 2002.

[7] M Mansha Ghalib, Azhar A Zafar, M Bilal Riaz, Z Hammouch, and Khurram Shabbir. Analytical approach for the steady mhd conjugate viscous fluid flow in 
a porous medium with nonsingular fractional derivative. Physica A: Statistical Mechanics and its Applications, page 123941, 2020.

[8] AK Gupta and S Saha Ray. Numerical treatment for the solution of fractional fifth-order sawada-kotera equation using second kind chebyshev wavelet method. Applied Mathematical Modelling, 39(17):5121-5130, 2015.

[9] Rizwan Ul Haq, Feroz Ahmed Soomro, Toufik Mekkaoui, and Qasem M AlMdallal. Mhd natural convection flow enclosure in a corrugated cavity filled with a porous medium. International Journal of Heat and Mass Transfer, 121:1168$1178,2018$.

[10] Heidar Hashemi, Zafar Namazian, Seyed Mohsen Hashem Zadeh, and SAM Mehryan. Mhd natural convection of a micropolar nanofluid flowing inside a radiative porous medium under ltne condition with an elliptical heat source. Journal of Molecular Liquids, 271:914-925, 2018.

[11] Tasawar Hayat, Tehseen Abbas, Muhammad Ayub, Taseer Muhammad, and Ahmed Alsaedi. On squeezed flow of jeffrey nanofluid between two parallel disks. Applied Sciences, 6(11):346, 2016.

[12] Asma Khalid, Ilyas Khan, Arshad Khan, and Sharidan Shafie. Influence of wall couple stress in mhd flow of a micropolar fluid in a porous medium with energy and concentration transfer. Results in Physics, 9:1172-1184, 2018.

[13] Thanon Korkiatsakul, Sanoe Koonprasert, and Khomsan Neamprem. New analytical solutions for time-fractional kolmogorov-petrovsky-piskunov equation with variety of initial boundary conditions. Mathematics, 7(9):813, 2019.

[14] Thanon Korkiatsakul, Sanoe Koonprasert, and Khomsan Neamprem. Chebyshev wavelet solutions for time-fractional integro partial differential equation and its application to beam problems. An international journal of advanced computer technology, 9(5):3677-3684, 2020.

[15] M Veera Krishna and Ali J Chamkha. Hall and ion slip effects on mhd rotating boundary layer flow of nanofluid past an infinite vertical plate embedded in a porous medium. Results in Physics, 15:102652, 2019.

[16] Liaquat Ali Lund, Zurni Omar, Ilyas Khan, Seifedine Kadry, Seungmin Rho, Irshad Ali Mari, and Kottakkaran Sooppy Nisar. Effect of viscous dissipation in heat transfer of mhd flow of micropolar fluid partial slip conditions: Dual solutions and stability analysis. Energies, 12(24):4617, 2019.

[17] Fazle Mabood and Kalidas Das. Outlining the impact of melting on mhd casson fluid flow past a stretching sheet in a porous medium with radiation. Heliyon, 5(2):e01216, 2019. 